\title{
The Role of Co-Enzyme Q10 in The Respiratory Chain and Some of Its Clinical Indications: A review
}

\author{
Noor Al-Huda A. Al-Zarqy, Zeina A. Al-Thanoon \\ College of Pharmacy, University of Mosul, Mosul, Iraq. \\ Corresponding author: noralhuda.php10@student.uomosul.edu.iq

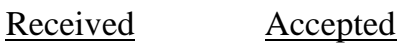 \\ 17-06-2021 31-07-2021
}

\begin{abstract}
Background: The usage of supplements becomes an important part of everyone health since many of these materials have produced real improvement in general health. These materials are not approved by organization like Food and Drug Administration in the US and the Food Safety Authority in Europe as drugs but their usage as supplement are approved globally. The supplements that will discuss in this review is co-enzyme Q10. It is a potent antioxidant and an essential part of respiratory chain. It acts as a mobile electron carrier between respiratory complexes. When co-enzyme Q10 transfer electron in the oxidative system, it acts to transport proton out of the mitochondria and this produce concentration gradient across membrane. Proton returns inside by enzymatic machine which involved ATP synthesis. Co-enzyme Q10 is the third wide-world used supplement. There are many studies confirm it benefit in many clinical conditions. These include the cardiovascular system, protection against statin induces myopathy, diabetes, neurodegenerative disease in addition to improvement in liver functions. These benefits occurred mainly due to its antioxidant effect and electron scavenging ability which result in reducing oxidative stress and related cell and tissue damage.
\end{abstract}

Objective This study tries to show the important role of coenzyme Q10 in energy production and spots the light toward the main clinical application of this supplement. Coenzyme Q10 is one of the main elements in the respiratory chain and it is endogenously synthesis since its presence is essential for life. Its availability may reduce in many disease conditions so supplementation of it within diet may become important in management of various disorders.

Keywords: Co-enzyme Q10, supplements, respiratory chain, mitochondrial bioenergetics, oxidative stress, protective effect.

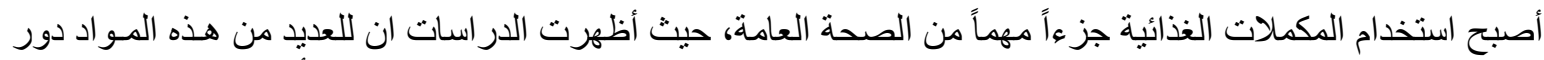

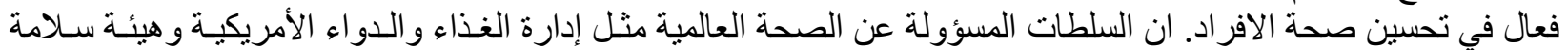

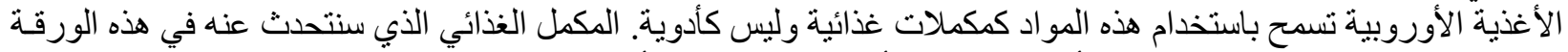

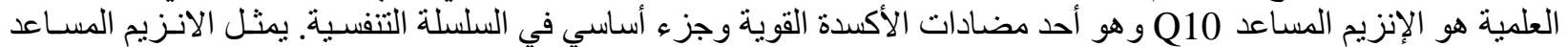

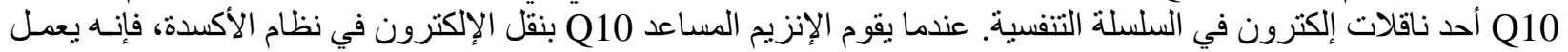

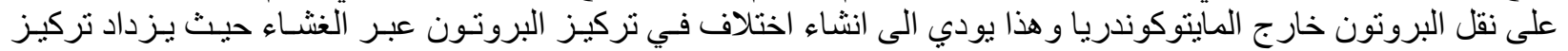

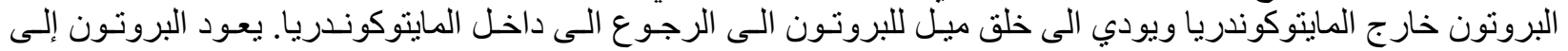

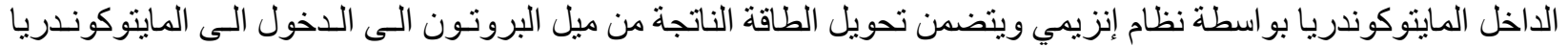

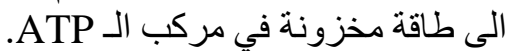


يعتبر الانزيم المساعد Q10 ثالث أكثر المكملات الغذائية المستخدمة عالميا. هناك العديد من الدر اسات التي تؤكد فعاليـة

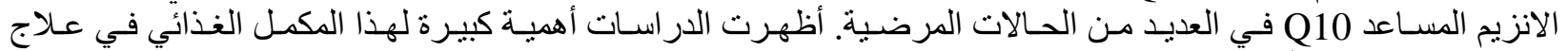

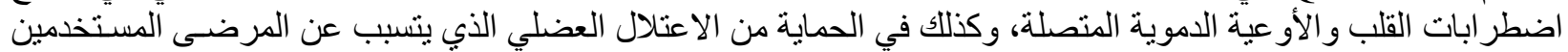

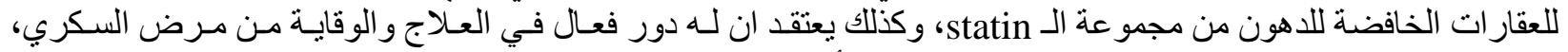

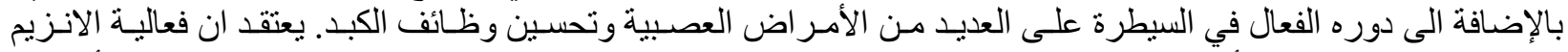

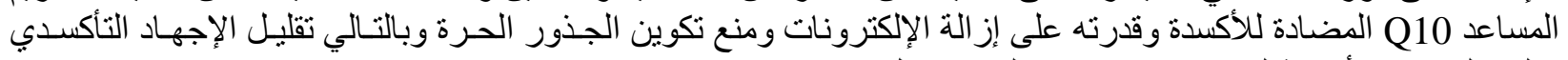
وتلف الخلايا والأنسجة لهم دور كبير في فعاليته كمكل غذأيدائي.

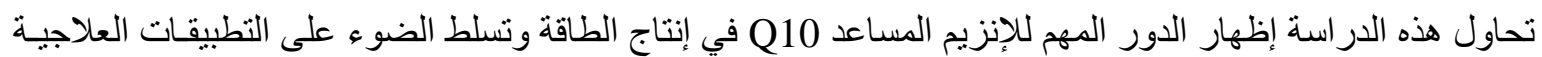

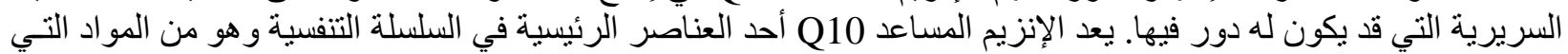

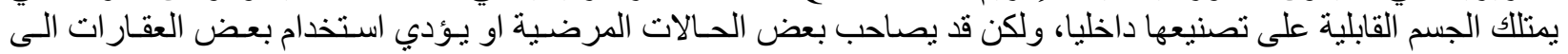

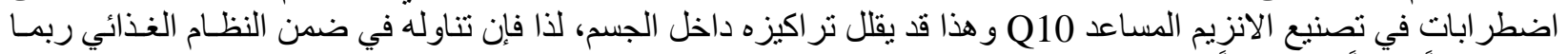
يودي دوراً وقائياً او علاجياً في العديد من الامر اض. فئ.

\section{Signification of supplements}

I $t$ is the unprecedented challenges that sustaining the health of more than nine billion people by 2050 . Researches in the nutrition and food areas are required to get solutions to these challenges which affect food systems and health. Indeed, one of the top of priorities for (WHO) World Health Organization is nutrition and food security. The enhancements in health as a result of increase health care in the last years have contributed to an improve in the life average, and this result in a remarkable increase of elderly worldwide (1).

When population getting old, this led to a harsh increase of the prevalence of noninfectious and chronic diseases. Cardiovascular diseases, such as hypertension, atherosclerosis, strokes had become the leading causes of death worldwide in the past decade, other noninfectious diseases including diabetes mellitus, cancer, dementias such as Alzheimer disease and others are in the list of the main 10 leading cause of death globally (1).

In truth, those are not only the main leading causes of death that produce killing in people more than all other causes combined, but they are also replacing malnutrition and communicable diseases as a cause of disability or early death. Although non-infectious diseases have become epidemic in their magnitudes, they can be prevented, in addition they could be reduced to significant extant by early detection, minimizing of their risk factors, and suitable management. In this spirit, governments are working to support lifestyles in healthy manner, and this to improve morbidity and mortality issues, and also to reduce the costs of health care (2).

Furthermore, knowledge about health, including applications related to food and fitness, make persons more alert about their own health's (3). Clients are now being more alert than any other time about the constituents in foods and the characteristic properties of these constituents, and by the increase number of clients who consider food as medication, food supplementation be obvious as one of the most rapid developing health related goods (4).

These in general have positive inferences in wellbeing condition, still, health related costs and the consuming growth are necessitating a critical need for physicians, specialized dietitians, and other regulators to get systematic data on safety and efficacy of a wide range of active constituents occurred in food supplements. Many clients think that supplements are natural occurring healthy foodstuffs with no possible interactions with drugs or 
contraindications. In fact, enormous of this health related products are having a hazard for a specific users (1).

For this cause, besides getting correct nutritional information from healthcare workers, users should be educated about the extent of available scientific information for particular indication of supplement, in addition, the interactions, precautions, and safe dose of the ingredients. For this reason, authorities like the European Food Safety Authority and the US Food and Drug Administration constantly perform efficacy and safety evaluations of constituents of supplement to dismiss or approve them $(3,5)$.

Conversely, more studies on pharmacokinetics, physical \& chemical characteristics, efficacy, and stability are required to expand the data about these constituents, and to enhance formulation by using appropriate technique. Suitable formulations can enhance supplements' bioavailability, and this can lead to greater efficacy also suitable formulation can improve stability (6-8).

When we talk about co-enzyme Q10, which is sold as a nutritional supplement and not as FDA-approved drug, it is represents $3^{\text {rd }}$ most used food supplement subsequent to omega 3 in fish oil and preparation contain multivitamins $(1,9)$.

It has strong antioxidant activity, and can be considered as an important part of physiological mitochondrial bioenergetics. In addition, it has been considered as an important choice for the management of various oxidative stress associated disorders such as neurodegenerative disorders, cardiovascular diseases and diabetes, which are in the top 10 main reasons of mortality $(10,11)$

\section{Coenzyme Q10 has important role in physiological mitochondrial bioenergetics}

Co-enzyme Q10 is an important element of cell mechanism used for generate ATP which acts to provide energy for almost all cellular functions. Co-enzyme Q10 exists in three redox states and this nature is essential for its function (12) :

* Fully oxidized ubiquinone (Co Q10)

* Fully reduced ubiquinol (Co Q10H2)

* Radical semiquinone intermediate (Co Q10H)

The production of ATP is occurred in the inner mitochondrial membrane, where a higher concentration of co-enzyme Q10 can be detected. The coenzyme Q10 has a distinctive role since it can not only transfer electrons from primary substrates to oxidative system but can, in the same time, transfer protons to the out of the mitochondrial. By this transfer, proton gradient across the mitochondrial membrane can generate and when the protons return to inside mitochondria by the enzymatic machine for ATP synthesis, they act as a method to ATP formation. The coenzyme Q10 bounds to the oriented enzymatic complexes. It acts to oxidize and release protons to the outside of mitochondria and picks up protons and electrons on the inside part of the mitochondrial membrane (12, 13).

There are five types of protein complexes in the mitochondrial membrane, two of them involved in protons and electrons transferred through coenzyme Q10. The first is known as the primary reductase where co-enzyme Q10 is reduced by the action of NADH this also known as (complex I). The reduction process involved four protons transport across membrane for every co-enzyme Q10 reduced $(12,13)$

The exact details about electron transport in (complex I) are still not fully understood, still, it has suggested that 
coenzyme Q10 is reduced and then reoxidized in (complex I) two times after that, electrons transferring to another lightly bound co-enzyme Q10 to produce ubiquinol which travels across the lipid of mitochondrial membrane to the another complex where ubiquinol oxidation occurred again in (complex III) and this happens with the transfer of protons against it concentration gradient across membrane $(12,13)$.

Unlike the details about protons transport in complex I, the details about ubiquinol oxidation and binding at the binding site of complex III are well known. Equal to complex I, there is oxidation, reduction and re-oxidation, also with the oxidation there is a proton release step to the outside so that protons released occurred in the right direction. Again oxidationreduction cycle permits four protons to pass the membrane for each ubiquinol oxidation cycle (12).
In a normal forward electron transfer chain, co-enzyme Q10 accepts electrons from both complexes I and II and transport them to complex III. In complex III, the Q cycle happened, with impelling of protons from the matrix to the intermembrane space. The complex III contains two distinct binding sites of coenzyme Q10. Reduced ubiquinol $\left(\mathrm{UQH}_{2}\right)$ bind at the $\mathrm{O}$ site (Qo site), transporting one electron to cytochrome $\mathrm{c}$ (cyt $\mathrm{c}$ ) and the other electron pass down to the I site (Qi site), where the electron attaches to coenzyme Q10 (ubiquinone), forming ubisemiquinone intermediate $\left(\mathrm{UQH}^{\circ}\right)$, or attach to $\left(\mathrm{UQH}^{*}\right)$ generated ubiquinol $\left(\mathrm{UQH}_{2}\right)$. The formation of oxidized coenzyme Q10 occurred at the (Qo site), while reduced ubiquinol $\left(\mathrm{UQH}_{2}\right)$ formed at the $(\mathrm{Qi}$ site). When electrons transport is occurred, they may leak out and attach to oxygen, forming superoxide anion $\left(\mathrm{O}_{2}{ }^{\circ-}\right)$ figure (1) (12).

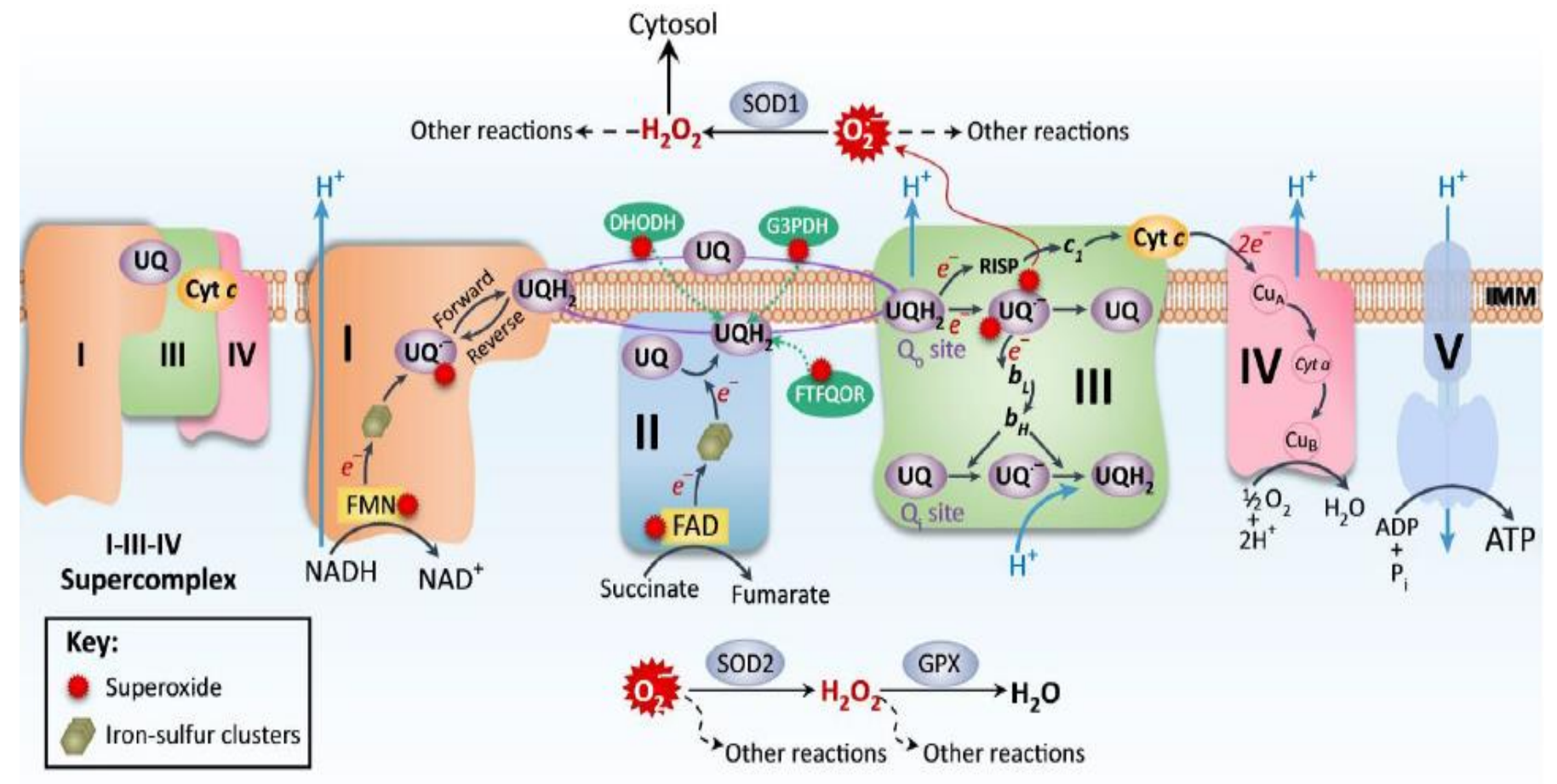

Figure 1 The functions of co-enzymeQ10 in the Respiratory Chain. Red stars indicate main sources of superoxide ( $\mathrm{O}^{2 \cdot-}$ ) production. (SOD) Superoxide dismutase, (GPX) glutathione peroxidase, $\left(\mathrm{O}^{2 \cdot-}\right)$ superoxide, $\left(\mathrm{H}_{2} \mathrm{O}_{2}\right)$ hydrogen peroxide, $\left(\mathrm{G}_{3} \mathrm{PDH}\right)$ mitochondrial glycerol-3-phosphate 
(DHODH)dehydrogenase dihydroorotate dehydrogenase, (ETFQOQ) electron transfer flavoprotein oxidoreductase. On the left of the figure, the I-III-IV super-complex is shown(12)

\section{Clinical application}

\section{Therapeutic effect of co-enzyme Q10 in Cardiovascular diseases}

Co-enzyme Q10 may have a potential advantage for subjects with cardiovascular disorders. Impaired mitochondrial function and subsequent oxidative stress has a fundamental effect in the pathophysiology of cardiovascular diseases (14). For this cause, in addition to its vital role in energy generation and its antioxidant effects, coenzyme Q10 could be considered as an auspicious candidate to prevent or management of cardiovascular disease. The review of Flowers et al. about the present data about the effect of coenzyme Q10 supplementation for the management of cardiovascular disorders indicate that despite that coenzyme Q10 has possible role for the management of current cardiovascular disorders, additional researches are required to determine whether it has an effect in the avoidance of such conditions in population have no current disorder (15). It has been noticed that low concentrations of coenzyme Q10 are linked with many disorders such as heart failure and cardiomyopathy (16). Coenzyme Q10 has an effect in the generation of energy, so its administration may have valuable properties in patients with low contractility of cardiac muscle owing to low energy condition that linked with coenzyme Q10 deficiency and result in heart failure (11).

In addition, the protective effect of coenzyme Q10 may be recognized because of its own useful effect on heart and circulation such as its desirable effect on atherosclerosis and hypertension (15). The proposed mechanism of antiatherosclerotic and antihypertensive may relate to its antioxidant effects.
In atherosclerosis, the antioxidant effect of coenzyme Q10 may diminish low density lipoprotein peroxidation (peroxidation of LDL) and improve endothelial function since the main marker for atherosclerosis is endothelial dysfunction so co-enzyme Q10 may act to improve this condition (17). While in oxidative stress conditions, there is a reduction in the availability nitric oxide and this lead to vasoconstriction and increase blood pressure, co-enzyme Q10 supplementation may preserve nitric oxide (18).

Despite a clinical trial that has been carried out, further researches with a larger sample size and a longer follow up duration has required to detect whether coenzyme Q10 has a protective effect in regard of atherosclerosis \& hypertension (19).

\section{Is coenzyme Q10 has role in the prevention of statins induced muscle problem/myopathy?}

Statins are a vital group of drugs that is applied in the management of dyslipidemia, so they have a role in the avoidance of cardiovascular disorders. Statins are in general safe drugs, although, a diversity of muscle problem/ myopathies have reported after its administration(20). Actually, about $10.5 \%$ of statins user may have myalgia, which is the main side effect of statin therapy (21). Even though, this is a minor side effect, it may affect patient's compliance.

The surveys among statin users suggest that about $30 \%$ of statin users discontinue their treatment because of weakness, pain in muscle, cramps, fatigue, and stiffness (22). In addition to these minor adverse effects, rhabdomyolysis a severe life threatening condition, may occur but it is extremely rare(23). 
The precise mechanism of this muscle problem/myopathy have not fully detected, but among suggested mechanisms, the effect on coenzyme Q10 de nova synthesis (24). The management of hypercholesterolemia with these drugs results in a low concentrations of co-enzyme Q10 (24, 25). Statins act by inhibiting HMG-CoA reductase, the enzyme that participate in regulation of the mevalonate pathway, the main precursor in the synthesis of many important substance include cholesterol and other agents include coenzyme Q10 (26). The consequence of coenzyme Q10 synthesis inhibition is compromise respiratory chain in the mitochondria and impairing production of energy and this may result in induction of myopathy (27).

Additionally, serum co-enzyme Q10 is carried by lipoproteins, statins-produce LDL reduction and that is another probable mechanism for co-enzyme Q10 depletion (28).

Statin therapy will discontinue according to patients complain if the possibility of rhabdomyolysis is presence. The supplementation of co-enzyme Q10 has recommended to treat and prevent myopathy induced by statin. In reality, there is a study that recommend the usage of supplemental coenzyme Q10 in all HMG-CoA reductase inhibitors users (29).Other studies reported that coenzyme Q10 supplementation in dose (30 to $200 \mathrm{mg} /$ day) may reduce muscle symptoms related to usage of statin, this may act as solution instead of discontinued treatment $(30,31)$. On the other hand, the result of meta-analysis study and systematic review cannot detect statistically significant beneficial effect of coenzyme Q10 administration in the improvement of myopathy induced by statin $(24,28)$.

The role of coenzyme Q10 in diabetic patients
Hyperglycemia is the main characteristic sign of diabetes, it has detected that the high extent of free radicals generation subsequence resultant oxidative stress, can play significant role in the pathophysiology of diabetes and diabeticrelated complications (32).

Actually, increase generation of free radicals associated with exhaustion of antioxidant defense can result in increased lipid peroxidation and cellular damage, this may lead to insulin resistance and diabetic complications (33). Coenzyme Q10 is one of the most potent antioxidants and it can act as a scavenger of free radical, so the assessment of its level can consider as an indicator of oxidative stress status within the body. High blood concentration of coenzyme Q10 had been reported not only in diabetic rats, but also in pediatrics with diabetes type 1 when their co-enzyme Q10 levels compared with healthy individuals $(34,35)$.

Despite this high blood levels, mitochondrial levels of coenzyme Q10 in liver \& heart of rats with diabetes are low. According to those, coenzyme Q10 can consider as a one of mechanisms that provide protection against oxidative stress condition. Moreover, many studies had performed to assess the promising positive effect of co-enzyme Q10 administration in diabetic patients. The complications of diabetic are linked to persist high levels of glucose, and that result in overproduction of superoxide anion in the mitochondria which acts to produce cellular and tissue damage by stimulate cell death and apoptosis (36). Cardiovascular complications are the major drawbacks of diabetes type 2 and it associated with malfunction of endothelium. When oxidative stress lead to the inhibit normal endothelial function, Many researches indicate, co-enzyme Q10 administration may enhance endothelial to produce their function properly by triggering 
mitochondrial oxidative phosphorylation and endothelial nitric oxide synthase(37).

\section{The effect of co-enzyme $Q \mathbf{1 0}$ in neurodegenerative disorders}

Mitochondrial dysfunction that produces abnormal energy metabolism, oxidative stress with its related increased oxidative damage and inflammation considers important etiologies for various neurodegenerative disorders.

The studying of antioxidant effect in the treatments of various condition is a site of interest (38). Co-enzyme Q10 is a mitochondrial function enhancer and strong antioxidant, so it could be a hopeful neuroprotectant to slow the progress of Parkinson's, Alzheimer's, and Huntington's diseases, along with other neurodegenerative disorders such as Friedrich's ataxia and amyotrophic lateral sclerosis $(10,39)$.

In actual fact, co-enzyme Q10 had shown a neuroprotective role in vitro, and that since it has stabilizing effect on the mitochondrial membrane of neuronal cells where they are exposed to oxidative stress. That result in to reduced cell death and/or damage, those are involved a cause of the abovementioned neurodegenerative disorders (40). In animal studies, the detected results confirm the neuroprotective effect of co-enzyme Q10 (41).

Actually, co-enzyme Q10 has the ability to defend neurons from injury by oxidation in a model of Parkinson's and (42) Alzheimer diseases, also it decreases generation of $\beta$-amyloid plaque in vivo a in mouse model with Alzheimer disease (43) and this lead to survival and behavioral enhancement in treated mouse with frontotemporal dementia (44).

In neurodegenerative diseases, there are association with low serum levels of coenzyme Q10, and that indicates poor antioxidant status, and a higher oxidative damage to cells and tissues.

Consequently, there is an indication for co-enzyme Q10 levels assessment to predict and follow up the development of neurodegenerative diseases, include dementia(45). Regardless of findings in animal models, that have confirmed a relationship between coenzyme Q10 and neuroprotection, until now, there are unsatisfactory evidences to support coenzyme Q10 usage routinely in humans with neuronal problems.

In the study of Chang et al., they established that since there are no clinical trials confirmed that coenzyme Q10 has been involved in the prevention of neurodegenerative disease progression or decreased their risk, this necessitates further clinical trials carried out in order to improve therapeutic strategies for these diseases (46).

\section{* Liver Disease}

Despite that endogenous coenzyme Q10 production take place in all the body, the liver is the main organ responsible for co-enzyme Q10 production this because of high metabolic ability in addition to large size of liver. In liver disease condition, the metabolic ability of liver has been decreased, and this produce lower levels of co-enzyme Q10. Low levels of coenzyme Q10 have possible harmful outcome on heart. So, subjects with liver diseases like nonalcoholic fatty liver disease (NAFLD) have risk factor for cardiovascular diseases. Cardiovascular diseases have described as main causes of death in subjects with NAFLD (47). Many cardiovascular problems are associated with NAFLD, these include arrhythmias, heart failure, atherosclerosis and valve dysfunction. In alcohol related liver disease, there is a similar association with an cardiovascular disorders and increase risk of many conditions like atrial fibrillation, alcoholic 
cardiomyopathy, and arterial hypertension (48).

In individuals with fatty liver disease who consuming statins, reduced concentrations of coenzyme Q10 may be a specific problem, and that in part because the inhibition of mevalonate pathway result in inhibition of cholesterol and co-enzyme Q10 synthesis. Coenzyme Q10 supplementation can act to reducing the of cardiovascular risks in subjects with liver disease, in addition it can produce liver benefit by decreasing oxidative stress and inflammation. It was expected that the main mechanisms of alcohol produces liver injury is by generate free radicals, and by the antioxidant action of co-enzyme Q10 protection of liver cells from such oxidative damage can occurred $(48,49)$.

Also free radicals can induce oxidative stress and this has been associated with the pathogenesis of NAFLD (50). Animal studies have confirmed the effect of coenzyme Q10 to prevent or reduce the progress of liver cirrhosis after exposure to different toxins including wide range of substance like toxic chemicals, drugs and even parasitic microorganisms $(48,51)$.

\section{References}

1. Arenas-Jal M, Suñé-Negre J, GarcíaMontoya E. Coenzyme Q10 supplementation: Efficacy, safety, and formulation challenges. Comprehensive Reviews in Food Science and Food Safety. 2020;19(2):574-94.

2. Kearney J. Food consumption trends and drivers. Philosophical transactions of the
One study to assess the effect of coenzyme Q10 supplement on liver toxicity done by the induction of liver damage in mice via administration of paracetamol and this followed by management with coenzyme Q10 to reduce the generation of cirrhosis tissue due to its anti-inflammatory and antioxidant effect. (50). In a similar study, in rats with a high risk to develop NAFLD, supplementation with coenzyme Q10 inhibited progression to cirrhosis via reduction of oxidative stress and inflammation(52). The studies established the ability of co-enzyme Q10 to protect liver against oxidative damage induced by free radical.

\section{Conclusion}

The aim of this review is to established the ability of co-enzyme Q10 to protect liver against certain of diseases such as cardiovascular, diabetes multiuse, neurological disorders and liver disease. The oxidative damage induced by free radical mechanism.

Conflict of interest: There is no conflict of interest.

royal society B: biological sciences. 2010;365(1554):2793-807.

3. Arenas-Jal M, Suñé-Negre J, PérezLozano P, García-Montoya E. Trends in the food and sports nutrition industry: A review. Critical reviews in food science and nutrition. 2020;60(14):2405-21.

4. Petrovic A, Hammer E. Are we positively or negatively affected?: A study 
on how people react to food-related communication messages. 2016.

5. EFSA Panel on Dietetic Products N, Allergies. Scientific Opinion on the substantiation of health claims related to coenzyme Q10 and contribution to normal energy-yielding metabolism (ID 1508, 1512, 1720, 1912, 4668), maintenance of normal blood pressure (ID 1509, 1721, 1911), protection of DNA, proteins and lipids from oxidative damage (ID 1510), contribution to normal cognitive function (ID 1511), maintenance of normal blood cholesterol concentrations (ID 1721) and increase in endurance capacity and/or endurance performance (ID 1913) pursuant to Article 13 (1) of Regulation (EC) No 1924/2006. EFSA Journal. 2010;8(10):1793.

6. Beg S, Javed S, JKohli K. Bioavailability enhancement of coenzyme Q10: an extensive review of patents. Recent patents on drug delivery \& formulation. 2010;4(3):245-57.

7. Kumar S, Rao R, Kumar A, Mahant $S$, Nanda S. Novel carriers for coenzyme Q10 delivery. Current drug delivery. 2016;13(8):1184-204.

8. Zhao X-H, Tang C-H. Spray-drying microencapsulation of CoQ10 in olive oil for enhanced water dispersion, stability and bioaccessibility: Influence of type of emulsifiers and/or wall materials. Food hydrocolloids. 2016;61:20-30.

9. Kapoor P, Kapoor A. Coenzyme Q10-a novel molecule. J Indian Acad Clin Med. 2013;14(1):37-45.

10. Dhanasekaran M, Ren J. The emerging role of coenzyme Q-10 in aging, neurodegeneration, cardiovascular disease, cancer and diabetes mellitus. Current Neurovascular Research. 2005;2(5):447-59.

11. Villalba JM, Parrado C, SantosGonzalez M, Alcain FJ. Therapeutic use of coenzyme Q10 and coenzyme Q10-related compounds and formulations. Expert opinion on investigational drugs. 2010;19(4):535-54.
12. Wang Y, Hekimi S. Understanding ubiquinone. Trends in cell biology. 2016;26(5):367-78.

13. Lipshutz BH, Mollard P, Pfeiffer SS, Chrisman W. A short, highly efficient synthesis of coenzyme Q10. Journal of the American Chemical Society. 2002;124(48):14282-3.

14. Chistiakov DA, Shkurat TP, Melnichenko AA, Grechko AV, Orekhov AN. The role of mitochondrial dysfunction in cardiovascular disease: a brief review. Annals of medicine. 2018;50(2):121-7.

15. Flowers N, Hartley L, Todkill D, Stranges S, Rees K. Co-enzyme Q10 supplementation for the primary prevention of cardiovascular disease. Cochrane Database of Systematic Reviews. 2014(12).

16. Molyneux SL, Florkowski CM, George PM, Pilbrow AP, Frampton CM, Lever M, et al. Coenzyme Q10: an independent predictor of mortality in chronic heart failure. Journal of the American College of Cardiology. 2008;52(18):1435-41.

17. Witting PK, Pettersson K, Stocker R. Anti-atherogenic effect of coenzyme Q10 in apolipoprotein E gene knockout mice. Free Radical Biology and Medicine. 2000;29(34):295-305.

18. Yang Y-K, Wang L-P, Chen L, Yao X-P, Yang K-Q, Gao L-G, et al. Coenzyme Q10 treatment of cardiovascular disorders of ageing including heart failure, hypertension and endothelial dysfunction. Clinica Chimica Acta. 2015;450:83-9.

19. Ayers J, Cook J, Koenig RA, Sisson EM, Dixon DL. Recent developments in the role of coenzyme Q10 for coronary heart disease: a systematic review. Current atherosclerosis reports. 2018;20(6):1-7.

20. Selva-O'Callaghan A, AlvaradoCardenas M, Pinal-Fernández I, TralleroAraguás E, Milisenda JC, Martínez MÁ, et al. Statin-induced myalgia and myositis: an update on pathogenesis and clinical 
recommendations. Expert review of clinical immunology. 2018;14(3):215-24.

21. Bruckert E, Hayem G, Dejager S, Yau C, Bégaud B. Mild to moderate muscular symptoms with high-dosage statin therapy in hyperlipidemic patients - the PRIMO study. Cardiovascular drugs and therapy. 2005;19(6):403-14.

22. Rosenbaum D, Dallongeville J, Sabouret P, Bruckert E. Discontinuation of statin therapy due to muscular side effects: a survey in real life. Nutrition, Metabolism and Cardiovascular Diseases. 2013;23(9):871-5.

23. Mendes P, Robles PG, Mathur S. Statin-induced rhabdomyolysis: a comprehensive review of case reports. Physiotherapy Canada. 2014;66(2):124-32.

24. Banach M, Serban C, Ursoniu S, Rysz J, Muntner P, Toth PP, et al. Statin therapy and plasma coenzyme Q10 concentrations - a systematic review and meta-analysis of placebo-controlled trials. Pharmacological research. 2015;99:329-36.

25. Qu H, Meng Y-y, Chai H, Liang F, Zhang J-y, Gao Z-y, et al. The effect of statin treatment on circulating coenzyme Q10 concentrations: an updated metaanalysis of randomized controlled trials. European journal of medical research. 2018;23(1):1-10.

26. Nawarskas JJ. HMG-CoA reductase inhibitors and coenzyme Q10. Cardiology in review. 2005;13(2):76-9.

27. Abd TT, Jacobson TA. Statininduced myopathy: a review and update. Expert opinion on drug safety. 2011;10(3):373-87.

28. Marcoff L, Thompson PD. The role of coenzyme Q10 in statin-associated myopathy: a systematic review. Journal of the American College of Cardiology. 2007;49(23):2231-7.

29. Langsjoen PH, Langsjoen AM. The clinical use of HMG CoA-reductase inhibitors and the associated depletion of coenzyme Q10. A review of animal and human publications. Biofactors. 2003;18(14):101-11.

30. Caso G, Kelly P, McNurlan MA, Lawson WE. Effect of coenzyme q10 on myopathic symptoms in patients treated with statins. The American journal of cardiology. 2007;99(10):1409-12.

31. Littlefield N, Beckstrand RL, Luthy KE. Statins' effect on plasma levels of Coenzyme Q10 and improvement in myopathy with supplementation. Journal of the American Association of Nurse Practitioners. 2014;26(2):85-90.

32. Wei W, Liu Q, Tan Y, Liu L, Li X, Cai L. Oxidative stress, diabetes, and diabetic complications. Hemoglobin. 2009;33(5):370-7.

33. Maritim A, Sanders a, Watkins Iii J. Diabetes, oxidative stress, and antioxidants: a review. Journal of biochemical and molecular toxicology. 2003;17(1):24-38.

34. Kucharska J, Braunova Z, Ulicna O, Zlatos L, Gvozdjakova A. Deficit of coenzyme Q in heart and liver mitochondria of rats with streptozotocin-induced diabetes. Physiological research. 2000;49(4):411-8.

35. Menke $T$, Niklowitz $P$, Wiesel $T$, Andler W. Antioxidant level and redox status of coenzyme Q10 in the plasma and blood cells of children with diabetes mellitus type 1. Pediatric diabetes. 2008;9(6):540-5.

36. Giacco F, Brownlee M. Oxidative stress and diabetic complications. Circulation research. 2010;107(9):1058-70.

37. Chew G, Watts G. Coenzyme Q10 and diabetic endotheliopathy: oxidative stress and the 'recoupling hypothesis'. Qjm. 2004;97(8):537-48.

38. Salama M, Yuan T-F, Machado S, Murillo-Rodríguez E, A Vega J, MenéndezGonzález M, et al. Co-enzyme Q10 to treat neurological disorders: basic mechanisms, clinical outcomes, and future research direction. CNS \& Neurological DisordersDrug Targets (Formerly Current Drug Targets-CNS \& Neurological Disorders). 2013;12(5):641-64. 
39. Mancuso M, Orsucci D, Calsolaro V, Choub A, Siciliano G. Coenzyme Q10 and neurological diseases. Pharmaceuticals. 2009;2(3):134-49.

40. Somayajulu M, McCarthy S, Hung M, Sikorska M, Borowy-Borowski H, Pandey S. Role of mitochondria in neuronal cell death induced by oxidative stress; neuroprotection by Coenzyme Q10. Neurobiology of disease. 2005;18(3):61827.

41. Spindler M, Beal MF, Henchcliffe C. Coenzyme Q10 effects in neurodegenerative disease. Neuropsychiatric disease and treatment. 2009;5:597.

42. Sharma S, Kheradpezhou M, Shavali $\mathrm{S}$, El Refaey H, Eken J, Hagen C, et al. Neuroprotective actions of coenzyme Q10 in Parkinson's disease. Methods in enzymology. 2004;382:488-509.

43. Yang $\mathrm{X}$, Dai G, Li G, Yang ES. Coenzyme Q10 reduces $\beta$-amyloid plaque in an APP/PS1 transgenic mouse model of Alzheimer's disease. Journal of molecular neuroscience. 2010;41(1):110-3.

44. Elipenahli C, Stack C, Jainuddin S, Gerges M, Yang L, Starkov A, et al. Behavioral improvement after chronic administration of coenzyme Q10 in P301S transgenic mice. Journal of Alzheimer's Disease. 2012;28(1):173-82.

45. Momiyama Y. Serum coenzyme Q10 levels as a predictor of dementia in a Japanese general population. Atherosclerosis. 2014;237(2):433-4.
46. Chang K-H, Cheng M-L, Chiang MC, Chen C-M. Lipophilic antioxidants in neurodegenerative diseases. Clinica Chimica Acta. 2018;485:79-87.

47. Francque SM, van der Graaff D, Kwanten WJ. Non-alcoholic fatty liver disease and cardiovascular risk: pathophysiological mechanisms and implications. Journal of hepatology. 2016;65(2):425-43.

48. Mantle D, Hargreaves I. Coenzyme Q10 and degenerative disorders affecting longevity: an overview. Antioxidants. 2019;8(2):44.

49. Mantle D, Preedy V. Free radicals as mediators of alcohol toxicity. Adverse drug reactions and toxicological reviews. 1999;18(4):235-52.

50. Spahis S, Delvin E, Borys J-M, Levy E. Oxidative stress as a critical factor in nonalcoholic fatty liver disease pathogenesis. Antioxidants \& redox signaling. 2017;26(10):519-41.

51. Fouad AA, Jresat I. Hepatoprotective effect of coenzyme Q10 in rats with acetaminophen toxicity. Environmental toxicology and pharmacology. 2012;33(2):158-67.

52. Tarry-Adkins JL, Fernandez-Twinn DS, Hargreaves IP, Neergheen V, Aiken CE, Martin-Gronert MS, et al. Coenzyme Q10 prevents hepatic fibrosis, inflammation, and oxidative stress in a male rat model of poor maternal nutrition and accelerated postnatal growth. The American journal of clinical nutrition. 2016;103(2):579-88. 